EPJ Web of Conferences 111,06001 (2016)

DOI: $10.1051 /$ epjconf/201611106001

(C) Owned by the authors, published by EDP Sciences, 2016

\title{
Neutron Cross Section Processing Methods for Improved Integral Benchmarking of Unresolved Resonance Region Evaluations
}

\author{
Jonathan A. Walsh ${ }^{1,2, a}$, Benoit Forget ${ }^{1}$, Kord S. Smith ${ }^{1}$, and Forrest B. Brown ${ }^{2}$ \\ ${ }^{1}$ Department of Nuclear Science \& Engineering \\ Massachusetts Institute of Technology \\ 77 Massachusetts Avenue, 24-107, Cambridge, MA, USA 02139
}

${ }^{2}$ XCP-3, Monte Carlo Methods, Codes, \& Applications

Los Alamos National Laboratory

Los Alamos, NM, USA 87545

\begin{abstract}
In this work we describe the development and application of computational methods for processing neutron cross section data in the unresolved resonance region (URR). These methods are integrated with a continuous-energy Monte Carlo neutron transport code, thereby enabling their use in high-fidelity analyses. Enhanced understanding of the effects of URR evaluation representations on calculated results is then obtained through utilization of the methods in Monte Carlo integral benchmark simulations of fast spectrum critical assemblies. First, we present a so-called on-the-fly (OTF) method for calculating and Doppler broadening URR cross sections. This method proceeds directly from ENDF-6 average unresolved resonance parameters and, thus, eliminates any need for a probability table generation pre-processing step in which tables are constructed at several energies for all desired temperatures. Significant memory reduction may be realized with the OTF method relative to a probability table treatment if many temperatures are needed. Next, we examine the effects of using a multi-level resonance formalism for resonance reconstruction in the URR. A comparison of results obtained by using the same stochastically-generated realization of resonance parameters in both the single-level Breit-Wigner (SLBW) and multi-level Breit-Wigner (MLBW) formalisms allows for the quantification of level-level interference effects on integrated tallies such as $k_{\text {eff }}$ and energy group reaction rates. Though, as is well-known, cross section values at any given incident energy may differ significantly between single-level and multi-level formulations, the observed effects on integral results are minimal in this investigation. Finally, we demonstrate the calculation of true expected values, and the statistical spread of those values, through independent Monte Carlo simulations, each using an independent realization of URR cross section structure throughout. It is observed that both probability table and OTF treatments reproduce the true expected values, calculated by averaging the results of many independent simulations, quite well. However, the spread of independent calculation results is shown to be relatively significant. The $k_{\text {eff }}$ eigenvalues for fast spectrum systems can differ by more than $250 \mathrm{pcm}$ from one simulation to the next.
\end{abstract}

\footnotetext{
ae-mail: walshjon@mit.edu
} 


\section{Introduction}

At the intermediate energies between the well-defined resonance structure of neutron cross sections in the resolved resonance region and the broad, smoothed fluctuations in the fast energy region, resonances do exist, but are so closely-spaced in energy that their individual parameters (i.e. resonance energy and partial widths) cannot be determined. A nuclide's unresolved resonance region (URR) is composed of the energies characterized by this phenomenon [1].

Treatment of the URR is of particular importance to the modeling of fast spectrum reactors and critical assemblies which often have a significant neutron flux in the URR of many of their constituent nuclides. Because simply using averaged cross sections is tantamount to neglecting energy self-shielding effects and results in over-predicted reaction rates, URR resonance structure must be accounted for. Implemented in many nuclear data processing codes such as NJOY [2], CALENDF [3], and here, as part of this work, the probability table method [4] is the most widely-used approach for incorporating this structure. Using the average URR resonance parameters contained in an ENDF-6 format [5] evaluation file and the theoretical distributions of those parameters, the method proceeds by generating many realizations of resonance structure and then tabulating the probabilities of observing various cross section magnitudes. This tabulation is performed at several URR energies for each desired temperature.

This work examines alternate methods for processing URR neutron cross sections and their use in integral benchmark simulations. Specifically, in Sec. 2, we start by presenting applications of an on-the-fly (OTF) cross section calculation method which eliminates the need for the pre-generation of probability tables. Next, in Sec. 3, the effects of using a multi-level resonance formalism for representing URR cross sections are analyzed. Then, in Sec. 4, the use of single, independent URR realizations throughout their own independent Monte Carlo simulations is investigated to test the ability of the probability table method to produce true expected value tallies and the spread of independent tally realizations. Lastly, concluding remarks are offered in Sec. 5.

All of the presented methods are implemented in the continuous-energy neutron transport code OpenMC [6]. Except where noted, results are obtained from OpenMC simulations utilizing ENDF/B-VII.1 nuclear data evaluations [7].

\section{On-the-Fly Cross Sections}

One drawback of the probability table method is that data must be pre-generated at multiple energies for each desired temperature. In simulations of systems with many temperatures (e.g. fast reactor multiphysics analyses), this can result in a large memory requirement. Calculating URR cross sections OTF (i.e. within the Monte Carlo transport calculation) directly from temperature-independent average resonance parameters can reduce this burden significantly [8]. As an additional benefit, the need for performing the sensitivity studies that are typically required to ensure that probability tables are generated with a fine enough phase-space mesh is also eliminated because the OTF method is continuous in the energy, temperature, and cross section magnitude variables.

Here, we use the OTF method to illustrate the testing of pre-generated probability tables and also demonstrate the significance of varying the URR energy bounds in simulations of the Zero Energy Breeder Reactor Assembly, core $8 \mathrm{H}$ (ZEBRA-8H) ${ }^{1,2}$. Fig. 1a shows the flux computed with both the OTF method and the probability tables of the ENDF71x ACE-format processed nuclear data library [11]. Agreement between the two flux spectra is an indication that the probability tables are gen-

\footnotetext{
${ }^{1}$ MIX-MET-FAST-008 from the International Criticality Safety Benchmark Evaluation Project (ICSBEP) [9].

${ }^{2}$ ZEBRA-FUND-RESR-001 from the International Reactor Physics Experiment Evaluation Project (IRPhEP) [10].
} 
erated with a phase-space mesh that is sufficiently fine to produce accurate tallies for the ECCO-33 energy group structure that is used in these simulations. Fig. $1 \mathrm{~b}$ plots the $k_{\infty}$ eigenvalues computed with varying URR upper energy bounds. The upward trend of $k_{\infty}$ with increasing upper bound suggests that resonance structure may be important at energies above the current ${ }^{238} \mathrm{U}$ ENDF/B-VII.1 URR evaluation. As further indication of this, simulations utilizing Fröhner's complete evaluation [12], up to $300 \mathrm{keV}$, result in $k_{\infty}$ eigenvalues that are $200 \mathrm{pcm}$ closer to the benchmark value than those computed using the truncated ENDF/B-VII.1 evaluation.

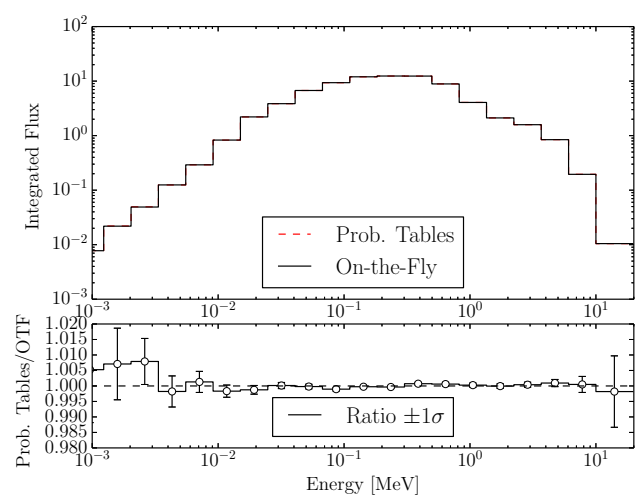

(a) Probability table mesh refinement studies

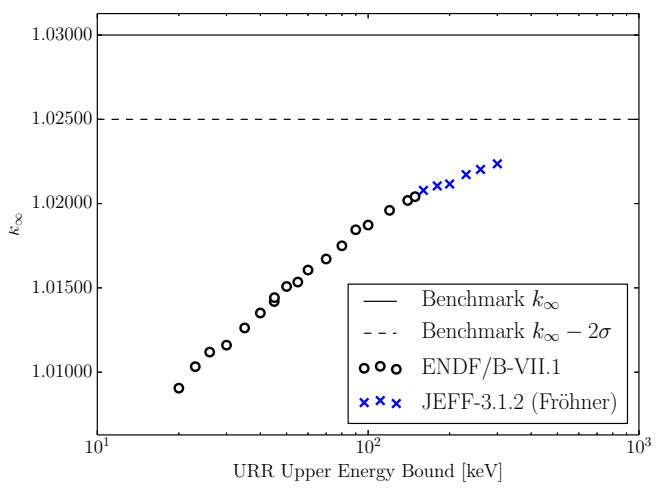

(b) Energy range sensitivity studies

Figure 1: URR evaluation benchmarking applications with ZEBRA-8H.

\section{Multi-Level Resonance Representation}

As is prescribed by the ENDF-6 format, the probability table method is typically implemented using the single-level Breit-Wigner (SLBW) resonance formalism to generate URR cross section realizations $^{3}$. Here, we investigate the use of the multi-level Breit-Wigner (MLBW) resonance formalism for OTF URR cross section calculations ${ }^{4}$ and compare the results to those obtained using the standard SLBW model in simulations of the Big Ten critical assembly ${ }^{5}$. Flux and ${ }^{238} \mathrm{U}$ scattering rate spectra for both formalisms are shown in Figs. $2 \mathrm{a}$ and 2b, respectively. Close agreement between the SLBW and MLBW spectra suggest that the use of a multi-level resonance formalism may not be necessary to reproduce many tallies of interest. This is further supported by the agreement between SLBW and MLBW $k_{\text {eff }}$ values that is seen in Table 1.

Table 1: Big Ten $k_{\text {eff }}$ eigenvalues.

\begin{tabular}{c|cccc}
\hline Method & ENDF71x prob. tables & OpenMC prob. tables & OTF SLBW & OTF MLBW \\
\hline$k_{\text {eff }}\left(1 \sigma \times 10^{5}\right)$ & $1.00467(10)$ & $1.00466(10)$ & $1.00461(10)$ & $1.00453(9)$ \\
\hline
\end{tabular}

\footnotetext{
${ }^{3}$ One exception is CALENDF which allows SLBW, multi-niveau Breit-Wigner, and Reich-Moore formalisms.

${ }^{4}$ In this preliminary study we investigate only the MLBW formalism as specified by the ENDF-6 format.

${ }^{5}$ IEU-MET-FAST-007, case 4 from the International Criticality Safety Benchmark Evaluation Project (ICSBEP) [9].
} 


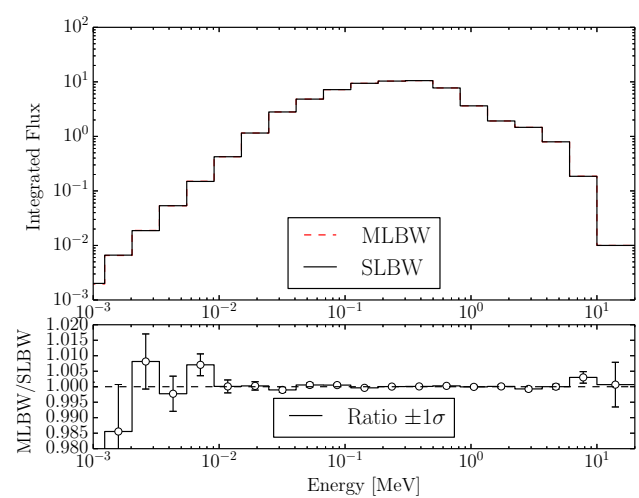

(a) Flux

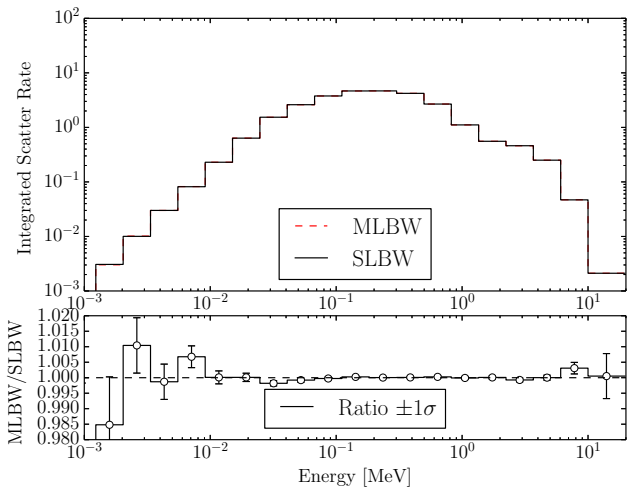

(b) ${ }^{238} \mathrm{U}$ scattering rate

Figure 2: SLBW and MLBW Big Ten tally spectra.

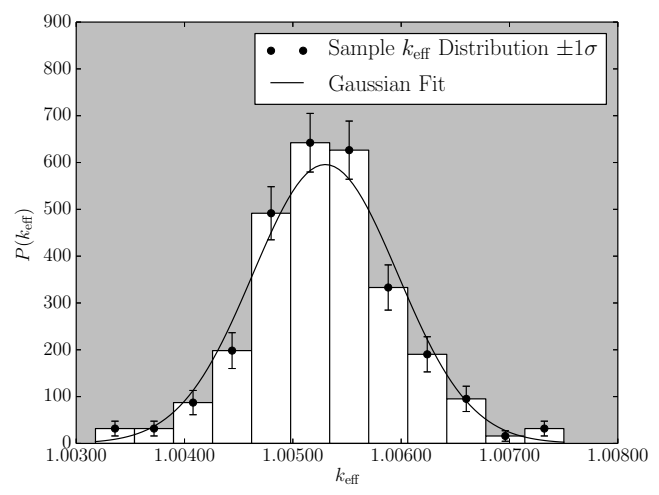

(a) $k_{\text {eff }}$ eigenvalues

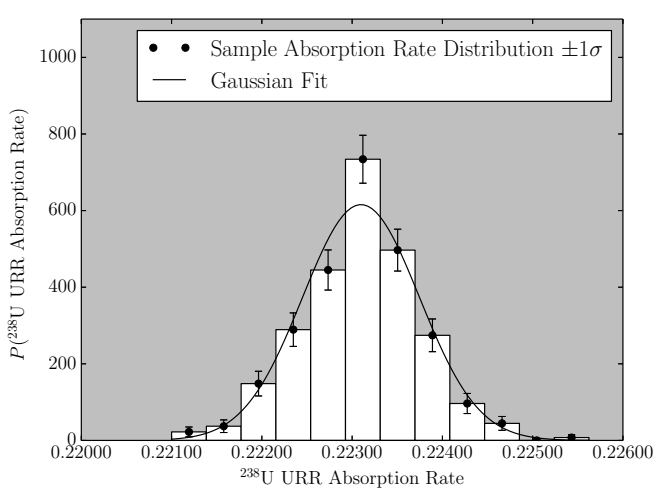

(b) ${ }^{238}$ U URR absorption rate

Figure 3: Big Ten tally distributions from independent realizations.

\section{Independent Resonance Realizations}

The typical probability table algorithm and the newly introduced OTF method both assume that neutrons, at a given energy, experience resonance structure that is independent of all structure previously encountered. The result is an artificial averaging over all possible resonance structures whereas, in nature, a single resonance structure exists. As a consequence, it is not guaranteed that simulations using probability tables or OTF calculations must reproduce true expected value tallies. However, by generating independent realizations of URR resonance structure and utilizing each of these realizations throughout its own independent Monte Carlo transport simulation, calculation of true expected value tallies is possible. Also, with each independent simulation now representing a viable realization of nature, it is possible to determine the spread of possible tally results. This spread represents the uncertainty induced by a lack of knowledge of precise URR resonance structure and cannot be ascertained using the probability table or OTF methods. 
The distributions of $k_{\text {eff }}$ and ${ }^{238}$ U URR absorption rate from 350 simulations of the Big Ten critical assembly are displayed in Figs. 3a and 3b, respectively. The centers of these distributions, which are the true expected values, agree very well with the results produced using the probability table and OTF methods ${ }^{6}$. However, the spread of individual tally results, each corresponding to a valid realization of nature, is notable with the middle $95 \%$ of $k_{\text {eff }}$ values spanning a range of $275 \mathrm{pcm}$.

\section{Conclusions}

This work demonstrates the use of various neutron cross section processing methods which allow for enhanced analyses of URR evaluations with integral benchmark simulations. An OTF cross section calculation approach is used to compute continuous phase-space results directly from resonance parameters. Further, it is used to demonstrate the variation of $k_{\text {eff }}$ with increasing URR upper energy bound. This variation indicates a possible need for modeling resonance structure at energies above the upper bounds of the ${ }^{238} \mathrm{U}$ URR evaluations that are currently available. The ENDF-6 version of the MLBW resonance formalism is also used to calculate OTF URR cross sections. Differences in both $k_{\text {eff }}$ and spectral tallies between simulations using the two formalisms are observed to be insignificant. Finally, independent simulations, each utilizing a single, independent URR realization, are used to compute true expected value tallies. The spread of tally realizations arising from unknown URR resonance structure is also demonstrated to be significant for a fast spectrum system with individual $k_{\mathrm{eff}}$ values showing differences greater than $250 \mathrm{pcm}$.

This work is funded by a U.S. Department of Energy (DOE) Nuclear Energy University Programs Graduate Fellowship, the Consortium for Advanced Simulation of Light Water Reactors (CASL), and the DOE National Nuclear Security Administration (NNSA) Advanced Simulation and Computing (ASC) program.

\section{References}

[1] A. Foderaro, The Elements of Neutron Interaction Theory (MIT Press, 1971)

[2] R. Macfarlane, D. Muir, Tech. Rep. LA-12740-M, Los Alamos National Laboratory (1994)

[3] J.C. Sublet, P. Ribon, M. Coste-Delclaux, Tech. Rep. CEA-R-6277, French Alternative Energies and Atomic Energy Commission (2011)

[4] L. Levitt, Nucl. Sci. Eng. 49, 450 (1972)

[5] A. Trkov, M. Herman, D. Brown, Tech. Rep. BNL-90365-2009 Rev.2, Brookhaven National Laboratory (2012)

[6] P.K. Romano, B. Forget, Ann. Nucl. Energy 51, 274 (2013)

[7] M. Chadwick, M. Herman, P. Obložinský, M. Dunn, Y. Danon, A. Kahler, D. Smith, B. Pritychenko, G. Arbanas, R. Arcilla et al., Nucl. Data Sheets 112, 2887 (2011)

[8] J.A. Walsh, B. Forget, K.S. Smith, B.C. Kiedrowski, F.B. Brown, Direct, On-the-fly Calculation of Unresolved Resonance Region Cross Sections in Monte Carlo Simulations, in Proc. MEC 2015, Nashville (2015)

[9] NEA Nuclear Science Committee, Tech. Rep. NEA/NSC/DOC(95)03, OECD NEA (2013)

[10] NEA Nuclear Science Committee, Tech. Rep. NEA/NSC/DOC(2006)1, OECD NEA (2014)

[11] J.L. Conlin, D.K. Parsons, S.J. Gardiner, M. Gray, A. Kahler, M.B. Lee, M.C. White, Tech. Rep. LA-UR-13-20137, Los Alamos National Laboratory (2013)

[12] F.H. Fröhner, Nucl. Sci. Eng. 103, 119 (1989)

\footnotetext{
${ }^{6}$ The difference between the OTF and true expected eigenvalues is $12 \mathrm{pcm}$ with a $10 \mathrm{pcm} 1 \sigma$.
} 
